\title{
Characteristics of electromechanical window in anesthetized rabbit models of short QT and long QT syndromes
}

\author{
Vudhiporn Limprasutr'1, Nakkawee Saengklub², Pradtana Meedech', \\ Anusak Kijtawornrat ${ }^{1,3}$ and Robert L. Hamlin 4 \\ ${ }^{1}$ Department of Veterinary Physiology, Faculty of Veterinary Science, Chulalongkorn University, Bangkok 10330, Thailand \\ ${ }^{2}$ Department of Physiology, Faculty of Pharmacy, Mahidol University, Bangkok 10400, Thailand \\ ${ }^{3}$ Research clusters: Research Study and Testing of Drug's Effect Related to Cardiovascular System in \\ Laboratory Animals, Chulalongkorn University, Bangkok 10330, Thailand \\ ${ }^{4}$ QTest Labs, LLC. 6456 Fiesta Dr., Columbus, Ohio 43235, USA
}

(Received April 10, 2017; Accepted July 6, 2017)

\begin{abstract}
The current regulatory guidelines recommend the use of QT interval to assess the risk of arrhythmogenic potential of new chemical entities. Recently, the electromechanical window (EMW), the difference in duration between electrical and mechanical systole, has been proposed as markers for druginduced torsades de pointes (TdP); however, data of EMW in short QT model are not available. This study aimed to characterize the EMW as a marker for drug-induced ventricular arrhythmias in anesthetized rabbit model of long QT syndrome type 2 (LQT2) and short QT syndrome (SQTS) infused with reference compounds known to lengthen or shorten QT intervals. After rabbits were anesthetized with isoflurane, body surface electrocardiograms and left ventricular pressure were recorded. The LQT2 was produced by intravenous infusion with dofetilide $(n=6)$, quinidine $(n=6)$ and sotalol $(n=6)$ whereas the SQTS was induced by intravenous escalating concentrations of nicorandil $(n=7)$, pinacidil $(n=5)$ and cromakalim $(\mathrm{n}=5)$. The EMW in anesthetized rabbits ranged from 1.3 to $53.3 \mathrm{msec}$. All three drugs known to lengthen QT intervals prolonged QT and QTcF interval while the EMW was markedly decreased to negative values. Pinacidil significantly produced QT and QTcF shortening and significantly abbreviated the EMW $(p<0.05)$. This study demonstrated that the EMW is associated with QT intervals $(p<0.001)$. It is negative in the presence of QT-prolonging drugs while it is more positive in the presence of QT-shortening drugs. The results suggest that the EMW in anesthetized rabbits can be used in drug safety evaluation in addition to the QT interval.
\end{abstract}

Key words: Electromechanical window, Long QT, Rabbit, Safety Pharmacology, Short QT

\section{INTRODUCTION}

Torsade de pointes (TdP) and ventricular fibrillation (VF) remain a major concern for drug discovery and safety evaluation. Since those arrhythmias are very rare, the arrhythmia might slip through the safety assessment test and result in very low incidence of death in post-marketing surveillance (Hondeghem, 2006). Due to experimental complexity of using TdP and VF as the end point, the duration of QT and QTc interval has been used as the surrogate end point for ventricular arrhythmias by the pharmaceutical companies and requires by regulatory agencies (Pugsley et al., 2008). However, the relationship between drug-induced arrhythmia either TdP or VF and the dura- tion of QT/QTc interval is very poor.

It has been suggested that the predictive value of several surrogate markers is better than the predictive value of single end point assessment (Pugsley et al., 2008). The EMW characterizes by the time difference between the end of electrical systole and the completion of ventricular relaxation (van der Linde et al., 2010). In healthy subjects the duration of cardiac electrical and mechanical activity are closely matched so that the electrical systole (equivalent to QT interval) ends earlier than the completion of contractile relaxation, creating a positive EMW. The EMW may be measured non-invasively by the time lag between the end of QT interval and the second heart sound (Boudoulas et al., 1982). A negative EMW has

Correspondence: Anusak Kijtawornrat (E-mail: akijtawornrat@gmail.com,kanusak@hotmail.com) 
been linked to increased mortality risk in various cardiac diseases such as the coronary artery disease and mitral valve prolapse (Boudoulas et al., 1981, 1982; Chambers and Ward, 1987; De Caprio et al., 1984).

Recently, an electromechanical window (EMW) has been proposed as an emerging biomarker for TdP liability in anesthetized dog and guinea pig model of acquired long QT syndrome type 1 (LQT1) (Guns et al., 2012a, 2012b; van der Linde et al., 2010). While EMW has been shown to predict arrhythmias in the model of LQT1, it has not been evaluated in the setting of long QT syndrome type 2 (LQT2) and short QT syndrome (SQTS). Therefore, the main purpose of this study was to evaluate the characteristics of EMW as a surrogate marker for drug-induced ventricular arrhythmias in anesthetized rabbit model of LQT2 and SQTS infused with reference compounds known to lengthen or shorten QT intervals. The hypotheses of this study were (1) the EMW is negative in rabbit model of LQT2 and (2) the EMW is increased from normal value in rabbit model of SQTS.

\section{MATERIALS AND METHODS}

\section{Animals}

This study was approved by the Institutional Animal Care and Use Committee of Faculty of Veterinary Science, Chulalongkorn University (protocol number 13310072). All animal procedures were conducted in accordance with the guidelines published in the Guide for the Care and Use of Laboratory Animals.

\section{Surgical procedure}

A total of 41 animals, distributed equally by sex, were used. All rabbits were weighed between 2 and $2.6 \mathrm{~kg}$. All rabbits were anesthetized with tiletamine/zolazepam (Zoletil, Virbac, France) $25 \mathrm{mg} / \mathrm{kg}$, intramuscularly. After tracheotomy and intubation, the depth of anesthesia was maintained by $1.5-2.5 \%$ isoflurane with $100 \%$ oxygen. Subsequently, transthoracic electrocardiogram was recorded. A high-fidelity micromanometer catheter (Millar Instruments, Houston, TX, USA) was retrogradely advanced into the left ventricle (LV) via right internal carotid artery to determine LV pressure (LVP) signal. Intravenous catheter was positioned in the right jugular vein for drug administration. All animals were allowed to stabilize for at least 20 min after finishing the instrumentation.

\section{Experimental protocol}

Following instrumentation and hemodynamic stabilization, the steady-state electrocardiographic (RR, PQ, QRS, and QT intervals) and LVP parameters were obtained as baseline values. In LQT2 model, groups of rabbits were each given of the following compounds: $0.1 \mathrm{~mL} / \mathrm{kg} / \mathrm{min}$ vehicle containing $0.1 \mathrm{M} \mathrm{HCl}$ in normal saline $(\mathrm{n}=6)$, $10 \mu \mathrm{g} / \mathrm{kg} / \mathrm{min}$ dofetilide $(\mathrm{n}=6), 3 \mathrm{mg} / \mathrm{kg} / \mathrm{min}$ quinidine $(\mathrm{n}=6), 2 \mathrm{mg} / \mathrm{kg} / \mathrm{min}$ sotalol $(\mathrm{n}=6)$. In SQT model, the drugs tested in order to shorten QT interval were nicorandil $(0.3,0.5$ and $1.0 \mathrm{mg} / \mathrm{kg} / \mathrm{min}, \mathrm{n}=7)$, pinacidil $(0.1,0.3$ and $1.0 \mathrm{mg} / \mathrm{kg} / \mathrm{min}, \mathrm{n}=5)$ and cromakalim $(0.001,0.003$ and $0.01 \mathrm{mg} / \mathrm{kg} / \mathrm{min}, \mathrm{n}=5$ ). All doses were selected from our previous publications because they are known to lengthen and shorten QT intervals (Kijtawornrat et al., 2006a, 2006b, 2010; Panyasing et al., 2010). All doses were infused intravenously over a period of $10 \mathrm{~min}$. The infusion was stopped as soon as TdP or VF started or end of the dose. All rabbits were euthanized at the end of experiment.

\section{Drugs}

Dofetilide (Pfizer, Groton, CT, USA) was dissolved in $0.9 \% \mathrm{NaCl}$ with the help of $0.1 \mathrm{M}$ hydrochloric acid to form a stock concentration of $0.1 \mathrm{mg} / \mathrm{mL}$. Quinidine hydrochloride, nicorandil and cromakalim (Sigma-Aldrich, St. Louis, MO, USA) were dissolved in 1\% DMSO. Sotalol (Sigma-Aldrich) was dissolved in $0.9 \% \mathrm{NaCl}$. Pinacidil (Sigma-Aldrich) was dissolved in $5 \%$ ethanol. Analyses of dosing solutions were not performed; however, each drug was administrated to rabbits within 60 min of preparation.

\section{Data analysis and statistics}

Data were obtained by using EMKA-IOX acquisition systems with sampling rates of $1000 \mathrm{~Hz}$ and analyzed by using ECG-auto software version 2.5.1.31 (EMKA Technologies, Falls Church, VA, USA). All ECG and LVP parameters were measured 9 min after each drug dose had been infused or $1 \mathrm{~min}$ before the occurrence of TdP or VF. TdP was defined as a polymorphic ventricular tachycardia where clear twisting of the QRS complexes around the isoelectric line was observed. The VF was defined as a very rapid, chaotic electrical impulses to the ventricle. The ECG intervals were measured in beats that originated from the sinoatrial node. Measurements were made from all cardiac cycle in $1 \mathrm{~min}$ and the average was used. The QT interval was the duration from the beginning of the Q wave to the end of T wave. The QT interval was corrected for heart rate by dividing the QT interval by the cube root of the preceding RR interval (Fridericia, 1920). The EMW was calculated as the following equation: EMW = QLVPend-QT. The duration of mechanical systole was expressed as the contraction time (ctrT). The 
Electromechanical window in anesthetized rabbits with LQT and SQT

ctrT refers to the time during which the ventricles contract and eject blood into the aorta and pulmonary artery. Data are presented as mean \pm standard error of mean (SEM) or medians (interquartile range, i.e $25^{\text {th }}-75^{\text {th }}$ percentile). In LQT2 model, comparisons between vehicle and test compounds were made using two-way ANOVA with repeated measure whereas in SQT model, the differences among escalating doses of drugs from baseline were evaluated by one-way ANOVA with repeated measure, and if indicated by a significant F-statistic, means were compared by Dunnett post-hoc analyses. Pearson and linear regression analysis were used to determine the relationship between EMW and QT interval. In all cases, $\mathrm{p}$ $<0.05$ was considered as statistically significant. Receiver operating characteristic (ROC) curve analysis was performed to determine the predictive power of QT, QTcF and EMW in rabbits receiving vehicle and QT-prolonging drugs. Area under the ROC curve (AUC) and confidence intervals were calculated using commercially available software. Parameters that yielded an AUC that were greater than 0.8 were considered to have a validated predictive value for the occurrence of TdP. The optimal cutoff values were determined using Youden indexes.

\section{RESULTS}

In general, high quality electrocardiograms and left ventricular pressure were obtained from all of the rabbits. All rabbits $(n=41)$ at baseline while they were anesthetized, the recorded heart rates ranged from 178 to 288 bpm, the PQ intervals were $63.3 \pm 1.4 \mathrm{msec}$, the QRS complexes were $50.6 \pm 1.2 \mathrm{msec}$, the QT intervals were $163 \pm 3 \mathrm{msec}$, the QTcF intervals were $257 \pm 4 \mathrm{msec}$ and the EMW were $21.8 \pm 1.8 \mathrm{msec}$.

\section{Effects of known QT-lengthening compounds on ECG and LVP parameters}

Example tracings showing the simultaneous electrocardiogram (ECG) and left ventricular pressure (LVP) recorded at baseline and $9 \mathrm{~min}$ after $10 \mu \mathrm{g} / \mathrm{kg} / \mathrm{min}$ dofetilide administration are shown in Fig. 1. Effects of agents known to lengthen QTc interval are shown as percent changes form baseline in Table 1. When compared to baseline and vehicle, quinidine and sotalol but not dofetilide lengthened RR interval (i.e. decreased HR) significantly $(p<0.05)$. All QT-prolonging drugs significantly prolonged PQ interval $(\mathrm{p}<0.05)$. Sotalol did not alter QRS complex while dofetilide and quinidine lengthened QRS complex significantly $(\mathrm{p}<0.05)$. Plots of EMW (Fig. 2A), QT (Fig. 2B) and QTcF (Fig. 2C) versus timepoints (before and after drug administration) are shown
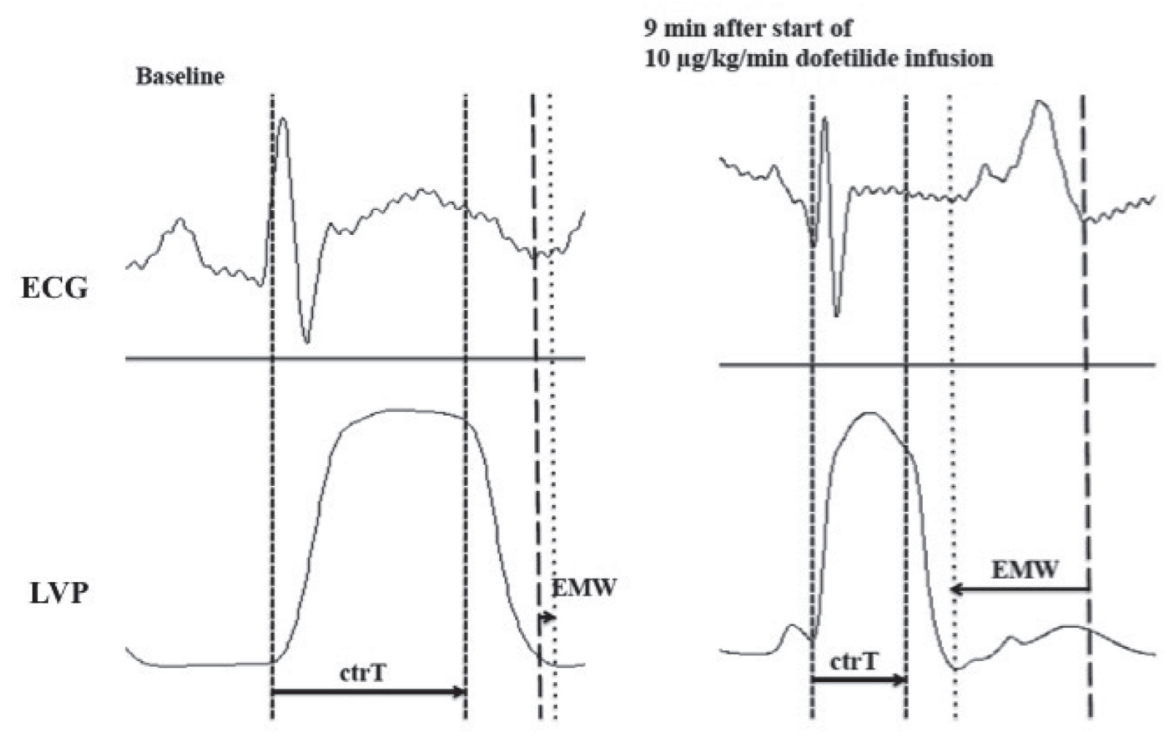

Fig. 1. Example tracings showing the simultaneous electrocardiogram (ECG) and left ventricular pressure (LVP) recorded at baseline and $9 \mathrm{~min}$ after $10 \mu \mathrm{g} / \mathrm{kg} / \mathrm{min}$ dofetilide administration. The duration between the dotted line (QLVPend) and the loosely dashed line (QT interval) is the electromechanical window (EMW). The duration between the densely dashed line is the contraction time (ctrT). 


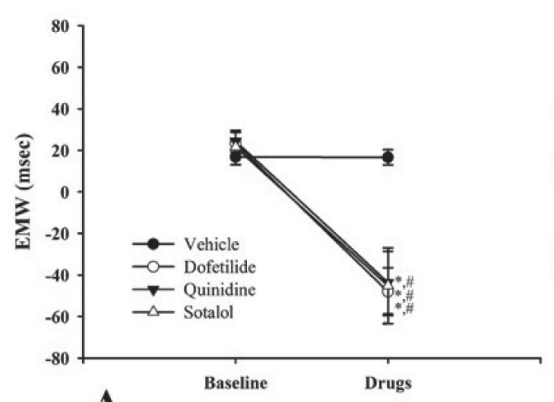

$\mathbf{A}$
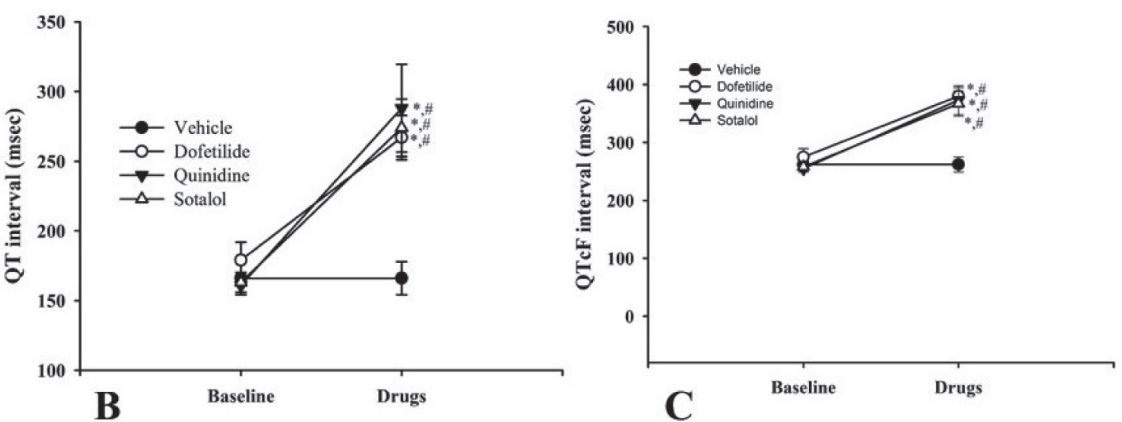

Fig. 2. Plots of mean and standard error of mean for electromechanical window (EMW, A), QT interval (B) and QTcF interval (C) versus timepoints (before and after drug administration). Rabbits were exposed to vehicle $(0.1 \mathrm{M} \mathrm{HCl}$ in $0.9 \% \mathrm{NaCl})$ or test articles known to lengthen QTcF interval (dofetilide, quinidine and sotalol). Each data point is the average of cardiac cycles for $1 \mathrm{~min}$. Doses of the reference compounds were: dofetilide, $10 \mu \mathrm{g} / \mathrm{kg} / \mathrm{min}$; quinidine, $3 \mathrm{mg} / \mathrm{kg} / \mathrm{min}$; and sotalol, $2 \mathrm{mg} / \mathrm{kg} / \mathrm{min}$. An asterisk $\left(^{*}\right)$ indicates $\mathrm{p}<0.05$ when a difference changed with statistical significance from baseline whereas \# indicates $\mathrm{p}<0.05$ when a difference changed with statistical significance from vehicle group.

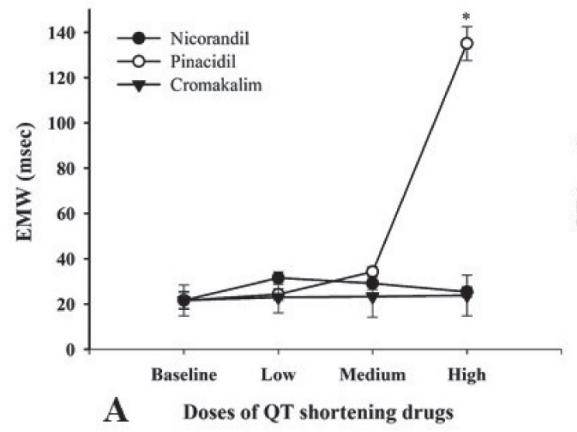

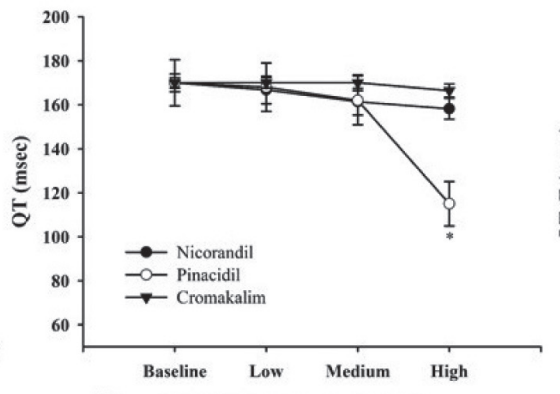

B Doses of QT shortening Drugs

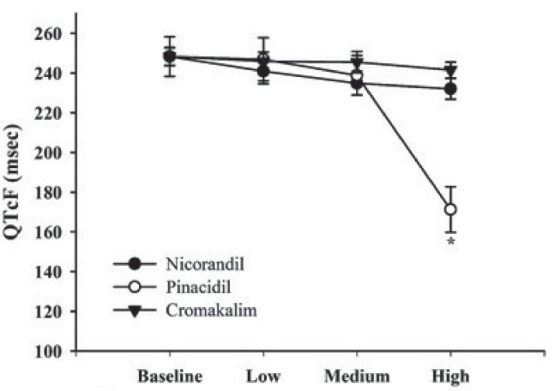

C Doses of QT shortening Drugs

Fig. 3. Plots of mean and standard error of mean for electromechanical window (EMW, A), QT interval (B) and QTcF interval (C) versus doses of QT-shortening drugs. Rabbits were exposed to escalating concentrations of test articles known to shorten QT interval (nicorandil, pinacidil and cromakalim). Each data point is the average of cardiac cycles for $1 \mathrm{~min}$. Doses of the reference compounds were: nicorandil, $0.3,0.5$ and $1.0 \mathrm{mg} / \mathrm{kg} / \mathrm{min}, \mathrm{n}=7$; pinacidil, $0.1,0.3$ and $1.0 \mathrm{mg} / \mathrm{kg} / \mathrm{min}$, $\mathrm{n}=5$; and cromakalim, $0.001,0.003$ and $0.01 \mathrm{mg} / \mathrm{kg} / \mathrm{min}, \mathrm{n}=5$. An asterisk $\left(^{*}\right)$ indicates $\mathrm{p}<0.05$ when a difference changed with statistical significance from baseline.

for groups of 6 rabbits each exposed to vehicle or test articles known to lengthen QTc interval. All test articles except vehicle significantly lengthened QT and QTcF intervals $(p<0.05)$ whereas the EMW were significantly shortened to negative values when compared to those values obtained at baseline or compared to those values obtained from rabbits receiving vehicle. Left ventricular pressure was also obtained in this study. As expected, quinidine and sotalol but not dofetilide decreased ESP, dP/ $\mathrm{dt}_{\max }$ and $\mathrm{dP} / \mathrm{dt}_{\min }$ significantly $(\mathrm{p}<0.05)$ when compared to baseline and vehicle (Table 1). However, EDP was not altered by any of QT-prolonging drugs. No significant change was detected for the ctrT among groups of rabbits receiving QT-prolonging drugs and vehicle.

\section{Effects of known QT-shortening compounds on ECG and LVP parameters}

Effects of agents known to shorten QTc interval are shown as percent changes from baseline in Table 2. The durations of RR, PQ and QRS were not affected by all three doses of nicorandil, pinacidil and cromakalim. Plots of EMW (Fig. 3A), QT (Fig. 3B) and QTcF (Fig. 3C) versus doses of QT-shortening drugs are shown. Only pinacidil markedly lengthened EMW and shortened QT and QTcF intervals when compared to those values obtained at baseline $(\mathrm{p}<0.05)$. All measured parameters of LVP (Table 2) were trivially changed in response to nicorandil and cromakalim. All three doses of pinacidil significantly reduced $\mathrm{ESP}, \mathrm{dP} / \mathrm{dt}_{\max }$ and $\mathrm{dP} / \mathrm{dt}_{\text {min }}(\mathrm{p}<0.05)$ when com- 
Electromechanical window in anesthetized rabbits with LQT and SQT


Vol. 42 No. 5 
pared with baseline. The ctrT was significantly increased in response to the highest dose of pinacil. No significant change was detected for the ctrT among groups of rabbits receiving nicorandil and cromakalim.

\section{Effects of test compounds on arrhythmia induction}

The incidence of drug-induced TdP or VF was also recorded. The TdP occurred 2 out of 6 rabbits (33.3\%) receiving intravenous infusion of dofetilide but not quinidine or sotalol. The VF occurred 1 out of 5 rabbits $(20 \%)$ receiving pinacidil administration but not nicorandil or cromakalim.

\section{Relationship between EMW and QT interval}

A plot of EMW versus QT interval for 41 anesthetized rabbits that received either vehicle or drugs known to lengthen QT (i.e. dofetilide, quinidine and sotalol) or shorten QT intervals (i.e. nicorandil, pinacidil and cromakalim) is shown in Fig. 4. The relationship between EMW and QT interval was EMW $=118-\left(0.6^{*} \mathrm{QT}\right)$, having $\mathrm{r}^{2}$ of 0.80 . This demonstrates that the relationship between the two is highly significant $(\mathrm{p}<0.001)$.

\section{Distribution of EMW in anesthetized rabbits}

A box-and-whisker plot showing the distribution of EMW values for anesthetized rabbits receiving vehicle,

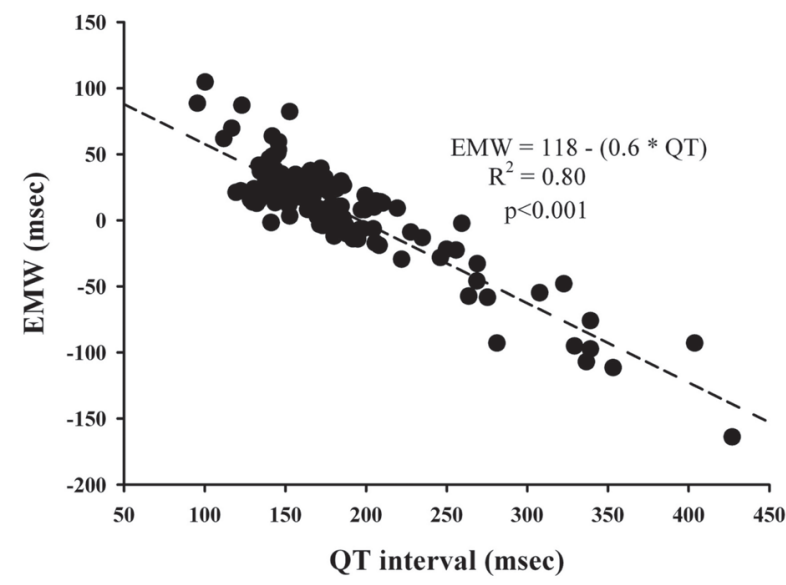

Fig. 4. Plot of averages of electromechanical window (EMW) versus QT interval for 41 anesthetized rabbits that received either vehicle $(0.1 \mathrm{M} \mathrm{HCl}$ in $0.9 \% \mathrm{NaCl})$ or drugs known to lengthen QT intervals (i.e. dofetilide, quinidine and sotalol) or known to shorten QT intervals (i.e. nicorandil, pinacidil and cromakalim). Notice that the relationship between EMW and QT interval was very high.
QT-prolonging drugs and QT-shortening drugs is provided in Fig. 5. The $25^{\text {th }}$ percentiles for vehicle, QT-prolonging drugs and QT-shortening drugs were 15.9, -75.8 and $26.3 \mathrm{msec}$ while the $75^{\text {th }}$ percentiles were $32.1,-19.1$ and $65.7 \mathrm{msec}$, respectively. The medians for those groups indicated by horizontal lines within the boxes were 23.6, -31.2 and $35.3 \mathrm{msec}$, respectively. There were no outliers in the data. There was statistically significant difference in the mean values of EMW among the groups (baseline vs QT-prolonging drugs, $\mathrm{p}<0.001$; baseline vs QT-shortening drugs, $\mathrm{p}<0.05)$.

\section{Predictive values of QT, QTcF and EMW in anesthetized rabbit model}

ROC analysis showed that both QT and QTcF intervals had AUC values less than 0.8 while the ROC analysis of the EMW yield AUC values greater than 0.8 (Table 3). Therefore, the QT and QTcF intervals were not valid predictive values for the occurrence of drug-induced TdP. The sensitivity of the EMW was 0.8 and the specificity of the EMW was 0.7 . Therefore, only EMW predicted drug-induced TdP with relatively high sensitivity and specificity. The optimal cut-off values of EMW was -32 msec (Fig. 6).

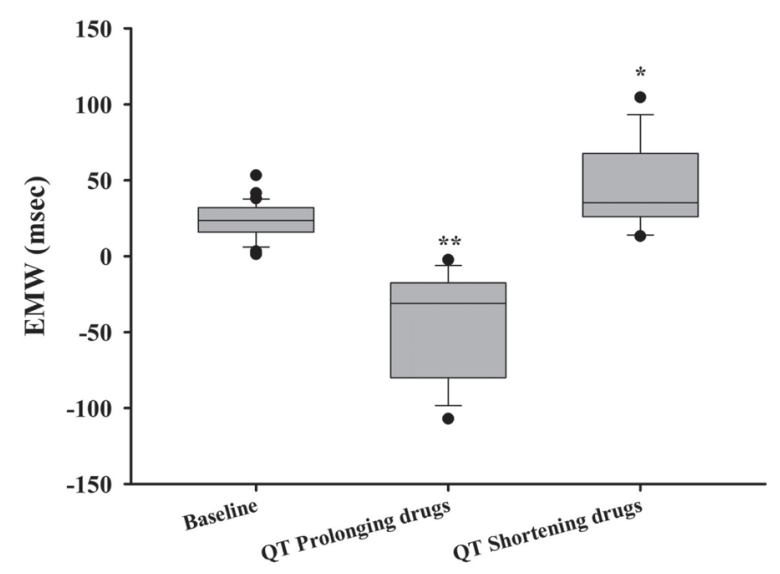

Fig. 5. A box-and-whisker plot showing the distribution of electromechanical window (EMW) values for baseline, QT-prolonging drugs and QT-shortening drugs. The boxes represent the $25^{\text {th }}$ to $75^{\text {th }}$ percentiles, and horizontal lines within the boxes indicate the medians. An asterisk $(*)$ indicates $\mathrm{p}<0.05$ and $(* *)$ indicates $\mathrm{p}<0.001$ compared with baseline data. 
Electromechanical window in anesthetized rabbits with LQT and SQT

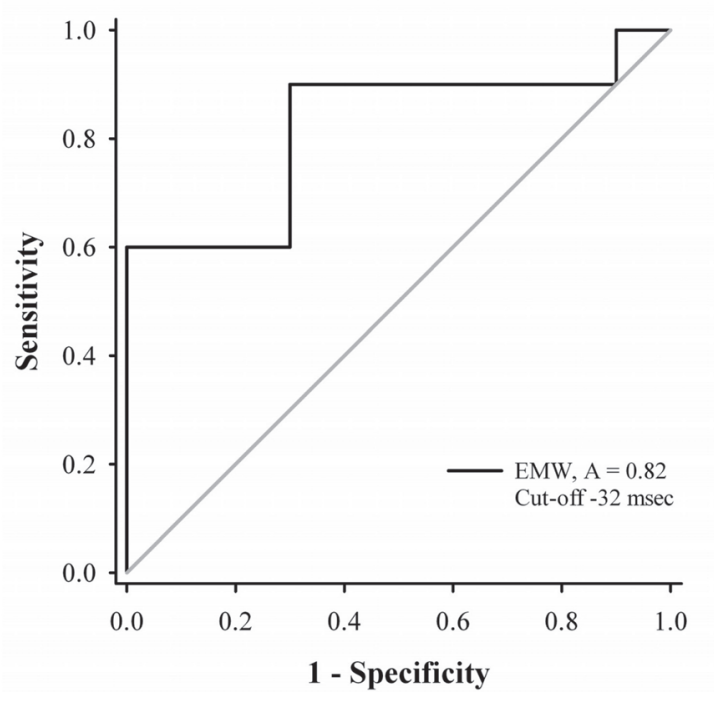

Fig. 6. Receiver operating characteristic (ROC) curve analysis of electromechanical window (EMW) for the prediction of dofetilide-induced torsades de pointes in anesthetized rabbits. The area under curve (AUC) is 0.82 while the optimal cut-off value of EMW was -32 msec.

Table 3. Area under the curve (AUC), sensitivity and specificity for QT, QTcF and EMW for prediction of dofetilide-induced TdP.

\begin{tabular}{lccc}
\hline Parameters & AUC & Sensitivity (\%) & Specificity (\%) \\
\hline QT & 0.69 & 70 & 45 \\
QTcF & 0.63 & 70 & 45 \\
EMW & 0.82 & 80 & 70 \\
\hline
\end{tabular}

AUC; area under the curve; QT; the QT interval; QTcF; the corrected QT interval by Fridericia's formula; EMW; electromechanical window

\section{DISCUSSION}

The main purpose of this study was to determine the characteristics of EMW in the anesthetized rabbit model of long QT and short QT syndromes. The results demonstrated that EMW shortened to negative values in rabbit model of long QT syndrome in response to all three reference compounds known to lengthen human QT intervals, and TdP was developed in dofetilide infusion. Interestingly, the EMW lengthened from baseline value in rabbit model of short QT syndrome in response to one of three reference agents known to shorten human QT intervals, and VF was developed in response to pinacidil administration.

In the present study, the EMW in anesthetized rabbits ranged from 1.3 to $53.3 \mathrm{msec}$. In response to QT-pro- longing drugs, the QT and QTcF intervals were increased while the EMW was decreased. This is in agreement with the finding of van der Linde and colleagues (2010) and Guns and colleagues (2012b), who reported that the negative EMW is required for drug-induced TdP in a long QT syndrome type 1. Furthermore, van de Linde suggested that the appearance of a negative EMW is a prerequisite to achieve a condition in which an R-on-T induces TdP. The author had observed $200 \mathrm{TdP}$ in their experiment by using a fentanyl/etomidate-anesthetized beagle dogs (van der Linde et al., 2010). In the present study, the incidence of TdP was $33.3 \%$ with dofetilide infusion. Although the EMW was reduced to a negative value and statistically significant from baseline values, the EMW values of rabbits that had TdP during dofetilide infusion were not statistically different from those values of rabbits that did not develop TdP while receiving dofetilide. This may partly due to the percent of rabbits developed TdP was small so that the power was not high enough to detect the differences between groups of rabbits. Interestingly, when the ROC analysis was performed to evaluate sensitivity and specificity of QT, QTcF and EMW between rabbits that develop TdP and rabbits that did not develop TdP while receiving dofetilide, only EMW had AUC value more than 0.8 with a sensitivity of $80 \%$ and specificity of $70 \%$. This indicated that EMW is superior to the QT and QTcF intervals for prediction of TdP in anesthetized rabbit model. However, since only dofetilide administration was able to induce TdP in this study the result of ROC analysis may be the limitation of this study. Further investigation must be performed to confirm this finding with other compounds that can produce TdP. On the other hand, previous study in halothane-anesthetized dogs (Izumi-Nakaseko et al., 2014) reported that the change of EMW was only due to the alteration of the QT interval since the duration of mechanical systole was not significantly changed in response to E-4031 administration. Therefore they concluded that the sensitivity of EMW to predict the onset of TdP in response to $\mathrm{I}_{\mathrm{Kr}}$ inhibitor was similar to the QT interval. The discrepancy between different conclusions among those studies may be due to the development of $\mathrm{TdP}$ since there is no TdP developed in the study of Izumi-Nakaseko and colleagues. This is consistent with previous report by Guns and colleagues (2012a), who found that drugs associated with prolongation of the QT interval and with well-documented TdP risk in humans (i.e. quinidine, terfenadine, dofetilide, haloperidol, domperidone and thioridazine) showed a dose-dependent prolongation of QT interval and shortening of EMW but did not evoke episodes of TdP when they were given to anesthetized guinea pigs. It has been known that blocking of both 
components (i.e. slow and rapid components) of delayed rectifier potassium channels produces markedly impaired repolarization reserve (Roden, 2008; Varró and Baczkó, 2011). In LQT1 model, blockades of the slowed component of delayed rectifier potassium channel $\left(\mathrm{I}_{\mathrm{Ks}}\right)$ (i.e. HMR1556, JNJ303) were used to trigger TdP (van der Linde et al., 2010; Guns et al., 2012b). Therefore, the difference of TdP episodes between the two models (LQT1 and LQT2) may be explained by severity of reduced repolarization reserve. The current study together with previous study (Izumi-Nakaseko et al., 2014) were similar in regards to the correlation between QT and EMW. In addition, both studies demonstrated that QT-prolonging drugs did not alter the duration of ventricular systole.

The current study demonstrated that the EMW in anesthetized rabbits receiving escalating doses of pinacidil was increased from baseline while the QT and QTcF intervals were shortened. This is in agreement with our previous study in conscious dogs in which the QT interval was shortened when dogs receiving all three reference compounds (Kijtawornrat et al., 2010). Although doses of nicorandil and cromakalim used in the current study were higher than previous study in dogs they failed to shorten QT interval in the anesthetized rabbits. This is may be due to the differences in pharmacokinetics and pharmacodynamics of drugs in different animal species (D'Alonzo et al., 1994a, 1994b; Horinaka et al., 2004). The finding of the current study showed that pinacidil induced VF in about $20 \%$ of the anesthetized rabbits. This is consistent with previous studies in which the use of ATP sensitive "openers" of potassium channel $\left(\mathrm{I}_{\mathrm{K} \text {-ATP }}\right)$ has been shown to be associated with VF (Chi et al., 1990; Lu et al., 2008; Padrini et al., 1992). It is known that $\mathrm{I}_{\mathrm{K} \text {-ATP }}$ openers shorten action potential duration so that the effective refractory period is abbreviated (Milberg et al., 2007). Pinacidil has also been shown to increase maximal transmural dispersion of repolarization between left and right ventricles in canine wedge preparation (Extramiana and Antzelevitch, 2004). These factors are known as substrates of arrhythmia mechanism (Antzelevitch and Burashnikov, 2011). In the present study, the sensitivity and specificity of QT, QTcF and EMW for prediction of VF were unable to analyze since the prevalence of pinacidile-induced VF was too low.

The use of conscious rabbit model and rabbits with heart diseases to test for torsadogenic compounds has been demonstrated previously (Kijtawornrat et al., 2006a, 2006b, 2012; Hamlin and Kijtawornrat, 2008). The present study also demonstrated the potential utility of the anesthetized rabbit for detecting the liability of test articles to lengthen or shorten QT/QTc intervals in humans.
Three reference compounds known to lengthen human QT intervals also prolonged QT intervals in the anesthetized rabbits; and one of three reference compounds known to shorten human QT intervals also abbreviated QT intervals in this model. There are several advantages of using rabbits to test for drug-induced prolongation and/or shortening of QT intervals. These advantages may include 1) the rabbit heart shares with humans all of the transmembrane ion channels specific for controlling ventricular repolarization (Romero et al., 2010) and 2) the calcium handling of human myocytes and rabbit ventricular myocytes was similar (Bers, 2002).

In conclusion, taken together, the results strongly indicated that the EMW is associated with the QT interval and it is negative in response to QT-prolonging drugs while it became more positive in response to QT-shortening drugs. In addition, the sensitivity and specificity of EMW for prediction of drug-induced TdP were in the acceptable values. Therefore, the EMW in anesthetized rabbits may be used as a marker for drug-induced TdP in response to administration of QT prolongation drugs.

\section{ACKNOWLEDGMENTS}

This study was supported by Chulalongkorn graduate scholarship to commemorate the $72^{\text {nd }}$ Anniversary of His Majesty King Bhumbol Adulyadej, the $90^{\text {th }}$ Anniversary of Chulalongkorn University Fund (Ratchadapiseksonphot Endowment Fund - grant number GCUGR1125572060D, Special Task Force for Activating Research (GSTAR 59-002-31-002). Dr.Vudhiporn Limprasutr was partly supported by the Graduate School, Chulalongkorn University (Overseas Research Experience Scholarship for Graduate Student) to perform research while she was at QTest Labs and the Support for the Overseas Presentations of Graduate Level Academic Thesis to present poster at Safety Pharmacology Society Annual Meeting.

Conflict of interest---- The authors declare that there is no conflict of interest.

\section{REFERENCES}

Antzelevitch, C. and Burashnikov, A. (2011): Overview of Basic Mechanisms of Cardiac Arrhythmia. Card. Electrophysiol. Clin., 3, 23-45.

Bers, D.M. (2002): Cardiac $\mathrm{Na} / \mathrm{Ca}$ exchange function in rabbit, mouse and man: what's the difference? J. Mol. Cell. Cardiol., 34, 369-373.

Boudoulas, H., Geleris, P., Lewis, R.P. and Leier, C.V. (1981): Effect of increased adrenergic activity on the relationship between electrical and mechanical systole. Circulation, 64, 28-33. 
Electromechanical window in anesthetized rabbits with LQT and SQT

Boudoulas, H., Sohn, Y.H., O'Neill, W., Brown, R. and Weissler, A.M. (1982): The QT greater than QS2 syndrome: a new mortality risk indicator in coronary artery disease. Am. J. Cardiol., 50, 1229-1235.

Chambers, J.B. and Ward, D.E. (1987): The QT and QS2 intervals in patients with mitral leaflet prolapse. Am. Heart J., 114, 355361.

Chi, L., Uprichard, A.C. and Lucchesi, B.R. (1990): Profibrillatory actions of pinacidil in a conscious canine model of sudden coronary death. J. Cardiovasc. Pharmacol., 15, 452-464.

D'Alonzo, A.J., Darbenzio, R.B., Hess, T.A., Sewter, J.C., Sleph, P.G. and Grover, G.J. (1994a): Effect of potassium on the action of the KATP modulators cromakalim, pinacidil, or glibenclamide on arrhythmias in isolated perfused rat heart subjected to regional ischaemia. Cardiovasc. Res., 28, 881-887.

D'Alonzo, A.J., Hess, T.A., Darbenzio, R.B., Sewter, J.C., Conder, M.L. and McCullough, J.R. (1994b): Effects of cromakalim or pinacidil on pacing- and ischemia-induced ventricular fibrillation in the anesthetized pig. Basic Res. Cardiol., 89, 163-176.

De Caprio, L., Ferro, G., Cuomo, S., Volpe, M., Artiaco, D., De Luca, N. and Ricciardelli, B. (1984): QT/QS2 ratio as an index of autonomic tone changes. Am. J. Cardiol., 53, 818-822.

Extramiana, F. and Antzelevitch, C. (2004): Amplified transmural dispersion of repolarization as the basis for arrhythmogenesis in a canine ventricular-wedge model of short-QT syndrome. Circulation, 110, 3661-3666.

Fridericia, L.S. (1920): Die Systolendauer im Elektrokardiogramm bei normalen Menschen und bei Herzkranken. Acta Med. Scand., 53, 469-486.

Guns, P.J., Johnson, D.M., Van Op den Bosch, J., Weltens, E. and Lissens, J. (2012a): The electro-mechanical window in anaesthetized guinea pigs: a new marker in screening for Torsade de Pointes risk. Br. J. Pharmacol., 166, 689-701.

Guns, P.J., Johnson, D.M., Weltens, E. and Lissens, J. (2012b): Negative electro-mechanical windows are required for drug-induced Torsades de Pointes in the anesthetized guinea pig. J. Pharmacol. Toxicol. Methods, 66, 125-134.

Hamlin, R.L. and Kijtawornrat, A. (2008): Use of the rabbit with a failing heart to test for torsadogenicity. Pharmacol. Ther., 119, 179-185.

Hondeghem, L.M. (2006): Thorough QT/QTc not so thorough: removes torsadogenic predictors from the T-wave, incriminates safe drugs, and misses profibrillatory drugs. J. Cardiovasc. Electrophysiol., 17, 337-340.

Horinaka, S., Kobayashi, N., Yabe, A., Asakawa, H., Yagi, H., Mori, Y., Tsubokou, Y., Yoshida, K., Nakano, S. and Matsuoka, H. (2004): Nicorandil protects against lethal ischemic ventricular arrhythmias and up-regulates endothelial nitric oxide synthase expression and sulfonylurea receptor $2 \mathrm{mRNA}$ in conscious rats with acute myocardial infarction. Cardiovasc. Drugs Ther., 18, 13-22.

Izumi-Nakaseko, H., Nakamura, Y., Cao, X., Ohara, H., Yamazaki,
Y., Ueda, N., Ando, K. and Sugiyama, A. (2014): Effects of selective IKr channel blockade by E-4031 on ventricular electromechanical relationship in the halothane-anesthetized dogs. Eur. J. Pharmacol., 740, 263-270.

Kijtawornrat, A., Nishijima, Y., Roche, B.M., Keene, B.W. and Hamlin, R.L. (2006a): Use of a failing rabbit heart as a model to predict torsadogenicity. Toxicol. Sci., 93, 205-212.

Kijtawornrat, A., Ozkanlar, Y., Keene, B.W., Roche, B.M., Hamlin, D.M. and Hamlin, R.L. (2006b): Assessment of druginduced QT interval prolongation in conscious rabbits. J. Pharmacol. Toxicol. Methods, 53, 168-173.

Kijtawornrat, A., Panyasing, Y., Del Rio, C. and Hamlin, R.L. (2010): Assessment of ECG interval and restitution parameters in the canine model of short QT syndrome. J. Pharmacol. Toxicol. Methods, 61, 231-237.

Kijtawornrat, A., Sawangkoon, S. and Hamlin, R.L. (2012): Assessment of QT-prolonging drugs in the isolated normal and failing rabbit hearts. J. Toxicol. Sci., 37, 455-462.

Lu, L., Reiter, M.J., Xu, Y., Chicco, A., Greyson, C.R. and Schwartz, G.G. (2008): Thiazolidinedione drugs block cardiac KATP channels and may increase propensity for ischaemic ventricular fibrillation in pigs. Diabetologia, 51, 675-685.

Milberg, P., Tegelkamp, R., Osada, N., Schimpf, R., Wolpert, C., Breithardt, G., Borggrefe, M. and Eckardt, L. (2007): Reduction of dispersion of repolarization and prolongation of postrepolarization refractoriness explain the antiarrhythmic effects of quinidine in a model of short QT syndrome. J. Cardiovasc. Electrophysiol., 18, 658-664.

Padrini, R., Bova, S., Cargnelli, G., Piovan, D. and Ferrari, M. (1992): Effects of pinacidil on guinea-pig isolated perfused heart with particular reference to the proarrhythmic effect. Br. J. Pharmacol., 105, 715-719.

Panyasing, Y., Kijtawornrat, A., Del Rio, C., Carnes, C. and Hamlin, R.L. (2010): Uni- or bi-ventricular hypertrophy and susceptibility to drug-induced torsades de pointes. J. Pharmacol. Toxicol. Methods, 62, 148-156.

Pugsley, M.K., Gallacher, D.J., Towart, R., Authier, S. and Curtis, M.J. (2008): Methods in safety pharmacology in focus. J. Pharmacol. Toxicol. Methods, 58, 69-71.

Roden, D.M. (2008): Cellular basis of drug-induced torsades de pointes. Br. J. Pharmacol., 154, 1502-1507.

Romero, L., Carbonell, B., Trenor, B., Rodriguez, B., Saiz, J. and Ferrero, J.M. (2010): Human and rabbit inter-species comparison of ionic mechanisms of arrhythmic risk: A simulation study. Conf. Proc. IEEE Eng. Med. Biol. Soc., 2010, 3253-3256.

van der Linde, H.J., Van Deuren, B., Somers, Y., Loenders, B., Towart, R. and Gallacher, D.J. (2010): The Electro-Mechanical window: a risk marker for Torsade de Pointes in a canine model of drug induced arrhythmias. Br. J. Pharmacol., 161, 1444-1454.

Varró, A. and Baczkó, I. (2011): Cardiac ventricular repolarization reserve: a principle for understanding drug-related proarrhythmic risk. Br. J. Pharmacol., 164, 14-36. 